Kiehne

Innerbetriebliche Standortplanung

und Raumzuordnung 
Schriften zur theoretischen und angewandten Betriebswirtschaftslehre

Herausgeber: Dr. Ludwig Pack

o. Professor der Betriebswittschaftslehre an der Universität Mannheim (WH)

Band 6 


\author{
Dr. Rolf Kiehne
}

\title{
Innerbetriebliche Standortplanung und Raumzuordnung
}


ISBN 978-3-663-00677-0 ISBN 978-3-663-02590-0 (eBook)

DOI 10.1007/978-3-663-02590-0

Verlags-Nr. 3115

Copyright by Springer Fachmedien Wiesbaden 1969

Ursprünglich erschienen bei Betriebswirtschaftlicher Verlag Dr. Th. Gabler GmbH, Wiesbaden 196

Softcover reprint of the hardcover 1st edition 1969 


\section{Vorwort des Herausgebers}

Das Standortproblem ist in der wirtschaftswissenschaftlichen Literatur bereits in vielfacher Weise behandelt worden. Soweit quantitative Verfahren zur Standortbestimmung beschrieben werden, betreffen sie jedoch fast ausschließlich die Bestimmung des optimalen Standortes eines Betriebes, eines Lagers oder einer Verkaufsstätte in einem größeren Wirtschaftsraum. Das Problem des optimalen innerbetrieblichen Standortes wird in der bisherigen Literatur meist rein deskriptiv in der Weise behandelt, daß die Faktoren beschrieben werden, welche über den innerbetrieblichen Standort die Höhe der innerbetrieblichen Transportkosten und den Arbeitsablauf beeinflussen.

Im Anschluß an eine exakte theoretische Fassung des innerbetrieblichen Standortproblems werden in der vorliegenden Arbeit die bisher in der Literatur zur quantitativen Lösung dieses Problems beschriebenen Verfahren dargestellt und miteinander verglichen. Die Leistungsfähigkeit dieser Verfahren unterliegt, was die Lösung praktischer Probleme der innerbetrieblichen Standortbestimmung anbetrifft, sehr bald erreichten Restriktionen. Diese Grenzen liegen sowohl in der Rechenzeit als auch in der Speicherkapazität der für die Anwendung dieser Verfahren notwendigerweise erforderlichen elektronischen Rechenanlagen.

Das in der vorliegenden Arbeit beschriebene Verfahren erlaubt auf einer EDV-Anlage des Typs IBM 7094 die Zuordnung von bis zu 10000 Einheiten (z. B'. Maschinen in einer Fabrikhalle, Angestellte in einem Büro usw.) in einer Rechenzeit von bis zu einer Stunde. Damit dürfte eine Größenordnung erreicht sein, welche rechentechnisch bedingte Restriktionen nicht mehr wirksam werden läßt. Die Schwierigkeiten, welche mit der Beschaffung der Daten für Probleme dieser Größenordnung in der für die Problemlösung zur Verfügung stehenden Zeit verbunden sind, dürften viel eher erreichte Grenzen setzen.

Das beschriebene Verfahren ist heuristischer Natur und führt nicht notwendigerweise zur besten aller möglichen Lösungen. Die praktische Anwendung des Verfahrens ergab jedoch in allen Testfällen Ergebnisse, die wesentlich besser waren als bei intuitiv vorgenommenen Standortbestimmungen. Der Mensch ist mit Problemen der Art, die mit den in dieser Arbeit beschriebenen Verfahren gelöst werden können, ganz einfach überfordert. Gibt es doch für die Verteilung von 10 Maschinen auf 10 mögliche Plätze bereits $10 !=3628800$ Möglichkeiten. Solange es, wie im vorliegenden Falle, noch keine Verfahren gibt, welche mit Sicherheit die optimale Lösung $\mathrm{zu}$ berechnen erlauben, besitzen unter diesen Umständen Verfahren, welche bessere Ergebnisse zeitigen als sie mit den bisher in der Praxis verwandten Methoden erzielt werden, bereits dann und auch dann große Bedeutung, wenn sie nicht mit Sicherheit zur besten aller möglichen Lösungen führen. 


\section{Inhaltsverzeichnis}

I. Einführung $\ldots \ldots \ldots \ldots \ldots \ldots$

1. Vorbemerkung zum Begriff des Systems . . . . . . . . . 1

2. Wirtschaftswissenschaftliche Standortfragen . . . . . . . . 2

3. Das Raumordnungsproblem ... . . . . . . . . . 6

4. Ziel, Aufgaben und Methodik der Untersuchung . . . . . . . 14

II. Modell und Modellgrößen . . . . . . . . . . . . . . . 19

1. Die allgemeine Formalisierung des Raumzuordnungsproblems . 19

2. Die spezielle Formalisierung des Raumzuordnungsproblems . . 25

3. Abgrenzung zu anderen Problemen ........... 31

4. Die Erfassung der Kontaktzahlen im Produktionsbereich . . . . 42

5. Die Erfassung der Kontaktzahlen in der Verwaltung . . . . . . 54

6. Die Aufteilung des Zuordnungsraums und ihre Konsequenzen für die Raumplanung . . . . . . . . . . . . 61

7. Die allgemeinen Fragen der Raumplanung . . . . . . . . . . 68

III. Lösungsmethoden und Lösungen - Voruntersuchung . . . . . . 72

1. Ansätze zur Lösung des normierten Raumzuordnungsproblems . 72

2. Übersicht über die speziellen Beispiele, mit deren Hilfe alle nachfolgenden Untersuchungen vorgenommen werden . . . 80

3. Die Verteilung der Zielwerte von Raumzuordnungsproblemen . 88

IV. Vertauschungsverfahren . . . . . . . . . . . 101

1. Die Auswirkung von Vertauschungen auf den Zielwert . . . . . 101

2. Zwei Iterationsverfahren auf Vertauschungsbasis . . . . . . 106

3. Suboptimale Lösungen . . . . . . . . . . . . . 110

4. Mögliche Änderungen des Schlußkriteriums . . . . . . . . . . 113

5. Einbettung in Bewegungsverfahren . . . . . . . . . . 116

6. Zur Abweichung suboptimaler Zielwerte vom Optimum . . . . . 120

7. Die durchgeführten Testrechnungen und deren Ergebnisse . . . 125

8. Möglichkeiten und Grenzen der Vertauschungsverfahren . . . . 134

9. Exkurs über die Berücksichtigung der Flächenbedarfe einzelner $\mathrm{OE}$ bei Vertauschungsverfahren - ein Vergleich mit einem Resultat von Armour . . . . . . . . . . . 138

V. Die Umlaufmethode . . . . . . . . . . . . . . . 145

1. Vorbemerkung über Verfahrenstypen . . . . . . . . . . 145

2. Grundzüge der Umlaufmethode . . . . . . . . . . . . 149

3. Modellhafte Ausprägung der Umlaufmethode . . . . . . . . 154

4. Programmierung des einfachen Umlaufverfahrens . . . . . . 157

5. Ergebnisse aus dem einfachen Umlaufprogramm und Vergleich mit dem Vertauschungsverfahren . . . . . . 168 
6. Speichertechnische Problematik der automatischen

Bearbeitung von Großproblemen . . . . . . . . . . . . . . . 174

7. Dichtspeicherung als Grundlage der ersten Ausbaustufe der Umlaufmethode . . . . . . . . . . . . . . . . 178

8. Die erste Ausbaustufe des Umlaufverfahrens -

Darstellung, Ergebnisse, Abgrenzung . . . . . . . . . . . . 189

9. Die zweite Ausbaustufe des Umlaufverfahrens -

Konzeption und Perspektiven . . . . . . . . . . . . . . . 198

V1. Die Kontraktionsmethode . . . . . . . . . . . . . . . . . 206

1. Die Konzeption der Kontraktion von Ahrens . . . . . . . . . 206

2. Kontraktion im n-dimensionalen Raum . . . . . . . . . . . . 210

3. Kontraktion als deterministischer Prozeß . . . . . . . . . . . 218

4. Kontraktion eines Resultates der Umlaufmethode . . . . . . . 229

VII. Die praktische Behandlung von Raumplanungsproblemen . . . . . 236

1. Zu einigen speziellen Raumplanungssituationen . . . . . . . . 236

2. Die Verteilung von organisatorischen Einheiten auf mehrere Stockwerke . . . . . . . . . . . . . . . . . . . 242

3. Die Anordnung innerhalb eines vorgegebenen Flächengrundrisses . . . . . . . . . . . . . 244

VIII. Zusammenfassung und Schluß . . . . . . . . . . . 250

Anhang: Vollständige Liste eines Rechenprogramms

für die Umlaufmethode . . . . . . . . . . . . . . . . . . . . . 253

Literaturverzeichnis . . . . . . . . . . . . . . . . 261 


\section{Verzeichnis der Tabellen}

Tabelle 1: Kontaktmatrix zu Beispiel (6a) . . . . . . . . . . . . 82

Tabelle 2: Kontaktmatrix zu Beispiel (6b) . . . . . . . . . . . . 82

Tabelle 3: Kontaktmatrix zu Beispiel (12) . . . . . . . . . . . 83

Tabelle 4: Häufigkeiten zu Beispiel (20) . . . . . . . . . . . . 84

Tabelle 5: Utbersicht über die auftretenden Beispiele und ihre Haupteigenschaften ...... . . . . . . 87

Tabelle 6: Zielwertverteilung der Beispiele (6a) und (6b) . . . . . . . . 92

Tabelle 7: Zielwertverteilung der Beispiele (12) bis (60) . . . . . . . . 95

Tabelle 8: Zufällige Zielwerte zu (194) und (341) . . . . . . . . . . . 100

Tabelle 9: Nach Vertauschungsverfahren berechnete Zielwerte für die Beispiele (12) bis (20/24) . . . . . . . . . . . . . . 127

Tabelle 10: Nach dem Vertauschungsverfahren II berechnete Zielwerte für die Beispiele (33) bis (60) . . . . . . . . . . . . 130

Tabelle 11: Rechenzeiten für die Vertauschungsverfahren bei den Beispielen mittlerer Größenordnung . . . . . . . . 131

Tabelle 12: Durch Vertauschungsverfahren erzielte Verbesserung . . . 131

Tabelle 13: Mittelpunktskoordinaten zu der Lösung in Abbildung 4 . . . 143

Tabelle 14: Hilfskoordinaten des Kleinumlaufs . . . . . . . . . . . 166

Tabelle 15: Ergebnisse des einfachen Umlaufverfahrens . . . . . . . 168

Tabelle 16: Rechnungsprotokoll zu (194) . . . . . . . . . . . . . . . . 191

Tabelle 17: Rechnungsprotokoll zu (341) . . . . . . . . . . . . . . 191

Tabelle 18: Zielwertevergleich . . . . . . . . . . . . . . 200 


\section{Verzeidhnis der Abbildungen}

Abb. 1: Wegenetze für die Beispiele (6a) und (6b) . . . . . . . . . . 90

Abb. 2: Zielwertverteilung der Beispiele (6a) und (6b) . . . . . . . . . 92

Abb. 3: Häufigkeitsverteilungen der Zielwerte . . . . . . . . . . 97

Abb. 4: Die ermittelte Lösung zu (20) mit Berücksichtigung

der Flächenbedarfe . . . . . . . . . . . . . . . . 142

Abb. 5: Die Umlaufwege bei verschiedenen 6-Kernen . . . . . . . 155

Abb. 6: a) Seitenvertauschung

b) Diagonalvertauschung . . . . . . . . . . . . 156

Abb. 7: Numerierung der Umlauffelder . . . . . . . . . . . 160

Abb. 8: Bildung des Anfangskerns . . . . . . . . . . . . . . 162

Abb. 9: Erste Numerierung der Umlauffelder . . . . . . . . . 162

Abb. 10: Teilraster zur Korrektur des Umlaufweges . . . . . . . . . . 164

Abb. 11: Charakteristische Umgebungssituationen für den Kleinumlauf . . . . . . . . . . . . . . . . . 165

Abb. 12: a) Lösung zu (12) aus Umlauf . . . . . . . . . . . . . . . . . 168

b) Lösung zu (20) aus Umlauf . . . . . . . . . . . . . . 169

Abb. 13: Kernumrisse zu den Umlauflösungen . . . . . . . . . . . . . 172

Abb. 14: Dichtspeichermethode für Beispiel (12) . . . . . . . . . 180

Abb. 15: Blockdiagramm des Suchprozesses . . . . . . . . . . . . . 181

Abb. 16: Blockdiagramm der Suchroutine . . . . . . . . . . . . . . 182

Abb. 17: Rechenzeitentwicklung während des Umlaufprozesses . . . . . . 196

Abb. 18: Kontraktionsergebnis zu (55) bei zufälliger Startlösung . . . . . 217

Abb. 19: Kontraktionsergebnis zu (55) mit einer guten Startlösung . . . . 231

Abb. 20: Ausgangslösung zum Kontraktionsergebnis in Abbildung 19 . . . 233 